\title{
Persistence of self-injurious behaviour in autism spectrum disorder over 3 years: a prospective cohort study of risk markers
}

\author{
Caroline Richards $^{1 *}$, Jo Moss ${ }^{1,2}$, Lisa Nelson ${ }^{1}$ and Chris Oliver ${ }^{1}$
}

\begin{abstract}
Background: There are few studies documenting the persistence of self-injury in individuals with autism spectrum disorder (ASD) and consequently limited data on behavioural and demographic characteristics associated with persistence. In this longitudinal study, we investigated self-injury in a cohort of individuals with ASD over 3 years to identify behavioural and demographic characteristics associated with persistence.

Methods: Carers of 67 individuals with ASD (Median age of individuals with ASD in years =13.5, Interquartile Range $=10.00-17.00$ ), completed questionnaires relating to the presence and topography of self-injury at $T_{1}$ and three years later at $T_{2}$. Analyses were conducted to evaluate the persistence of self-injury and to evaluate the behavioural and demographic characteristics associated with persistence of self-injury.

Results: At $\mathrm{T}_{2}$ self-injurious behaviour had persisted in $77.8 \%$ of individuals. Behavioural correlates of being non-verbal, having lower ability and higher levels of overactivity, impulsivity and repetitive behaviour, were associated with self-injury at both time points. Risk markers of impulsivity $(p=0.021)$ and deficits in social interaction $(p=0.026)$ at $T_{1}$ were associated with the persistence of self-injury over 3 years.

Conclusions: Impulsivity and deficits in social interaction are associated with persistent self-injury in ASD and thus may act as behavioural risk markers. The identification of these risk markers evidences a role for behaviour dysregulation in the development and maintenance of self-injury. The findings have clinical implications for proactive intervention; these behavioural characteristics may be utilised to identify 'at risk' individuals for whom self-injury is likely to be persistent and therefore those individuals for whom early intervention may be most warranted.
\end{abstract}

Keywords: Autism spectrum disorder, Self-injury, Risk marker, Prevalence, Hyperactivity, Impulsivity, Pain

\section{Background}

Self-injurious behaviour is common in autism spectrum disorder (ASD), with estimates ranging from 35 to $60 \%$ [1-5]. These prevalence estimates are significantly higher than those reported for populations with intellectual disability of heterogeneous aetiology [6-8]. The presence of self-injury leads to a higher risk of psychiatric hospitalisation [9], reactive physical intervention [10] and lower quality of life [11]. Carers and staff of those who display self-injury are reported to experience higher levels of stress and burnout [12-14]. In addition

\footnotetext{
* Correspondence: c.r.richards@bham.ac.uk

'Cerebra Centre for Neurodevelopmental Disorders, School of Psychology,

University of Birmingham, Edgbaston B15 2TT, UK

Full list of author information is available at the end of the article
}

to the personal costs of self-injury, there are also significant financial costs to services [15]. Behavioural interventions for self-injury, which employ function-based differential reinforcement procedures, are effective but are often resource intensive [16-18]. Consequently, attention has turned to the viability of a targeted early intervention strategy for self-injurious behaviour $[19,20]$, which is predicated on the assumption that self-injury begins during childhood/early adulthood, becomes more severe with time and persists over time [21-23].

Estimates of the persistence of self-injury in individuals with intellectual disabilities vary considerably. However, the majority of studies demonstrate that self-injury can be persistent in individuals with 
intellectual disability with large longitudinal studies reporting $71 \%$ persistence over 7 years (age range at follow-up $=12-65$ years) [23] and $84 \%$ persistence over 20 years (age range at follow-up $=24-82$ years) [22]. However, there are also a number of studies which appear to show some remission in self-injury. Murphy and colleagues demonstrated that the prevalence of self-injury significantly decreased over time (age range at follow-up $=13.5-30.4$ years) [24]. Similarly, Cooper and colleagues reported a moderate two year remission rate in adults with intellectual disability (38.2\%; mean and $S D$ age in years at follow-up =43.6; 14.2) [25]. However, despite presenting evidence that selfinjurious behaviour may not be as persistent as initially thought, the data reported by Cooper and colleagues reveal that $61.8 \%$ of adults continued to show self-injurious behaviour that caused tissue damage, was pervasive, presented significant risks to the health or safety of the person and significantly impacted upon their own or other's quality of life. Thus, these data still suggest that for the majority of individuals, self-injury continues to be a behaviour which significantly and persistently impacts upon their lives.

Despite the high prevalence of self-injury in ASD, few studies have examined persistence. A recent literature review suggests that challenging behaviours may be stable in individuals with ASD [26]. Cross-sectional designs offer convergent evidence that prevalence of selfinjury remains constant across subsamples of young children, children and young adolescents with ASD [27]. However, in studies with longitudinal designs of adolescents and adults with ASD [5] and children with pervasive developmental disorders (PDD) [28], the presence of self-injury decreased significantly over 4.5 and 3 years, respectively. However, both studies recruited through service agencies, clinics and hospitals where individuals were receiving treatment. Therefore, it is not possible to evaluate whether the reported remission in self-injury in ASD is the natural course of development or a result of intervention. Additionally, the sample recruited by Baghdadli and colleagues were young (mean age at follow-up 8 years), and thus, selfinjury behaviour may not yet have become established. A 7-year follow-up of the cohort described by Baghdadli and colleagues [2], reported that $35.8 \%$ of the sample showed self-injury, a similar prevalence figure to that reported originally by Baghdadli and colleagues (32.7\%). Whilst persistence was not evaluated statistically in the 7-year follow-up, the comparable prevalence figures in the two studies suggest that self-injury may have persisted. Thus, there remains a need to evaluate statistically the persistence of self-injury in a population with ASD that have not been recruited from clinical services. If persistence is high, then in combination with models of the development and maintenance of self-injury this would allude to the importance of early intervention.

Proactive intervention strategies to reduce negative outcomes have been implemented effectively in physical health settings [29] and more broadly in early intensive behavioural interventions for autism [30-32]. It is hoped that by providing interventions for socially maintained self-injury when individuals with ASD are young, outcomes will be more positive as reinforcement history for self-injury will be shorter and, consequently, the behaviours will be less resistant to change [21]. In order for early intervention strategies to be effective, it would be beneficial to identify those individuals with the greatest risk of developing self-injury that is likely to be persistent as opposed to transient. Delineating behavioural correlates (characteristics associated with the presence of self-injury at a single time point) and risk markers (characteristics associated with the persistence of self-injury over time) could aid early intervention thorough strategic targeting of interventions towards those who evidence the risk markers for persistent selfinjury at an earlier stage in the development of the behaviour.

There is emerging evidence of demographic and behavioural characteristics that are associated with selfinjury in ASD at a single time point. These putative risk markers include impairments in adaptive skills [1-3], greater severity of autism [1, 2, 33], younger age [34], perinatal conditions [1] and repetitive and impulsive behaviours [3, 4]. Studies of populations of individuals with intellectual disabilities have identified lower ability [25], lower verbal ability [35], attention deficit hyperactivity disorder [25], visual impairment [25] and the site of selfinjury $[23,35]$ as variables which independently predict the persistence of self-injury over time. Two studies have evaluated the predictive value of variables to identify persistent self-injury in ASD [5, 28]. Speech deficits, autism severity, intellectual disability and older age were all identified as risk markers. However, these studies were not able to link these risk markers to persistent selfinjury as self-injury was either grouped into an outcome subscale with repetitive, withdrawn and inattentive behaviours [5] or both onset of self-injury and persistence of self-injury were grouped into a 'negative outcome' category [28]. Thus, there remains a need to identify risk markers associated with persistent self-injury in individuals with ASD.

In summary, the prevalence of self-injury has been reliably demonstrated to be elevated in those with ASD compared to those with intellectual disability of heterogeneous aetiology $[1,3,5,36]$. There is an evidence that self-injury is persistent in the majority of individuals with intellectual disability; however, the 
data in individuals with ASD are equivocal $[5,23]$. Prior to a consideration of early intervention and putative risk markers for self-injury in ASD, evidence should be gathered regarding the persistence of selfinjury. In order to guard against threats to external validity, these data should be drawn from a population with ASD that has not been recruited from a clinical sample. There is evidence associating a range of demographic and behavioural characteristics with self-injury in ASD at a single time point $[1,34]$ and predicting persistent self-injury in intellectual disability populations $[23,25,35]$. However, there is currently very limited study in ASD of characteristics associated with self-injury at multiple time points and associated with persistent self-injury [5, 28]. These data could contribute to a targeted early intervention strategy. In this study, we conduct longitudinal assessments of a sample of individuals with ASD. A sub-group of this sample at the first time point of this study were reported by [3]. At the first time point, self-injury was associated with significantly higher levels of impulsivity, hyperactivity and negative affect and significantly lower levels of adaptive ability and speech. The present follow-up study has these aims:

i) To compare prevalence, topographies and severity of self-injury at $\left(T_{1}\right)$ and $\left(T_{2}\right) 3$ years later, to establish the persistence of self-injury.

ii) To investigate behavioural and demographic variables associated with self-injury at $\mathrm{T}_{2}$. We predicted that certain demographic and behavioural variables associated with self-injury in the longitudinal sample at $T_{1}$ and/or in previous literature (poor speech, impulsivity, overactivity, repetitive behaviours, autism spectrum disorder phenomenology) will also be associated with self-injury at $\mathrm{T}_{2}$.

iii) To evaluate the value of these behavioural and demographic variables at $T_{1}$ to differentiate between absent, transient and persistent self-injury at $\mathrm{T}_{2}$.

\section{Methods}

\section{Recruitment}

Participants with ASD were recruited in the UK via the National Autistic Society at $T_{1}$. These participants were contacted and invited to participate 3 years later at $\mathrm{T}_{2}$. In total, 190 participants were invited to take part at $\mathrm{T}_{2}$, and 68 carers of individuals with ASD completed the assessments (return rate $35.78 \%$ ). The average follow-up time was 36.4 months (range $=34-39$ months).

\section{Procedure}

Carers received an information sheet, cover letter, consent form, demographic questionnaire and questionnaire pack. To avoid priming, the study was described as investigating behaviours associated with ASD. Carers returned completed questionnaires and consent forms in a prepaid envelope.

Ethical approval for this study was obtained from the ethical review committee at the University of Birmingham

\section{Participants}

Participants were excluded if at $T_{1},(1)$ they were under the age of four as some measures were not appropriate, (2) they did not have a confirmed diagnosis of ASD from a relevant professional (Psychiatrists, Clinical Psychologists, Educational Psychologists, General Practitioner, Clinical Geneticist or Paediatrician) as reported by parents/carers, (3) they had an additional diagnosis of a comorbid genetic syndrome, (4) they had incomplete total scores on the Social Communication Questionnaire (SCQ) [37], and (5) they scored below the ASD cutoff on the SCQ. If a large proportion of the data at $\mathrm{T}_{1}$ or $\mathrm{T}_{2}$ were incomplete ( $25 \%$ or more of items across questionnaires), participants were also excluded. Thus, one participant was excluded from the sample.

A sub-group of the $T_{1}$ sample was reported on by Richards and colleagues [3]. This sub-group comprised only those who had an associated intellectual disability, defined by proxy via a score of less than nine on the self-help subscale of the Wessex [38]. However, in order to ensure a large and representative sample at follow-up, the whole sample (those with a score of less than nine and those with a score of nine on the self-help subscale) was invited to take part at $\mathrm{T}_{2}$.

To ensure that the $T_{2}$ sample was representative of the $\mathrm{T}_{1}$ sample, and not biased by 122 participants who declined to take part $\mathrm{T}_{2}$, a series of Mann-Whitney $U$ and $x^{2}$ analyses were conducted to detect possible significant differences between participants included at $T_{2}(67)$ and those from the $\mathrm{T}_{1}$ sample who were not included. Table 1 describes the demographic and behavioural characteristics of those who took part at $\mathrm{T}_{2}$ and those who declined to take part at $\mathrm{T}_{2}$

The analysis revealed that significantly more individuals without self-injury took part at $\mathrm{T}_{2}$ than individuals with self-injury. Apart from this, individuals who took part at $\mathrm{T}_{2}$ did not differ on any other demographic or behavioural variable, to the individuals who declined to take part at $T_{2}$. This suggests that the data sample collected at $\mathrm{T}_{2}$ is broadly representative of the original sample collected at $\mathrm{T}_{1}$.

\section{Measures}

The questionnaire pack included the following informant based questionnaire measures which are appropriate for children and adults with intellectual disabilities. 
Table $1 T_{1}$ Demographic characteristics comparing participants at $T_{2}$ with those who declined to participate at $T_{2}$

\begin{tabular}{|c|c|c|c|c|c|c|}
\hline$N$ & & $\begin{array}{l}\text { Took part at } T_{2} \\
67\end{array}$ & $\begin{array}{l}\text { Declined to take part at } T_{2} \\
122\end{array}$ & Mann-Whitney U/X & $d f$ & $p$ value $^{\mathrm{a}}$ \\
\hline Age & Median (IQR) & $10.00(7.00-14.00)$ & $10.00(7.00-14.00)$ & 4287.50 & - & 0.576 \\
\hline Gender & $\%$ male & 85.1 & 86.1 & 0.35 & 1 & 0.852 \\
\hline Self help & $\%$ partly able/able & 89.6 & 85.2 & 0.70 & 1 & 0.403 \\
\hline Mobility & $\%$ mobile & 98.5 & 95.1 & $N / A^{c}$ & - & 0.425 \\
\hline Speech & $\%$ verbal & 89.6 & 82.8 & 1.30 & 1 & 0.255 \\
\hline Self-injury & $\%$ with behaviour & 40.3 & 54.9 & 3.92 & 1 & 0.048 \\
\hline Mood $^{d}$ total score & Median (IQR) & $34.00(30.00-38.00)$ & $32.00(27.00-38.00)$ & 4470.00 & - & 0.287 \\
\hline Activity ${ }^{e}$ total score & Median (IQR) & $44.00(24.63-53.00)$ & $44.00(30.00-56.00)$ & 3566.00 & - & 0.172 \\
\hline Repetitive behaviour total score & Median (IQR) & $28.00(17.00-37.00)$ & $29.50(17.10-40.00)$ & 3748.00 & - & 0.443 \\
\hline ASD phenomenology ${ }^{9}$ total score & Median (IQR) & $28.00(23.00-31.00)$ & $26.00(22.00-30.12)$ & 3722.5 & - & 0.310 \\
\hline
\end{tabular}

${ }^{\mathrm{a}}$ Significant differences are highlighted in italics $(p<0.05$; two tailed)

${ }^{b}$ Based on the self-help scale of the Wessex Behaviour Schedule. Able or partly able is defined as a score of $>2$

'Fishers exact was calculated as two cells had an expected count of $<5$

${ }^{\mathrm{d}}$ Mood, Interest and Pleasure Questionnaire

'The Activity Questionnaire

fRepetitive Behaviour Questionnaire

${ }^{\mathrm{g} S o c i a l}$ Communication Questionnaire

A demographic questionnaire to collect information on gender, mobility, verbal ability and diagnosis.

The Wessex [38] was used to assess ability in children and adults with intellectual disabilities. It comprises five subscales including continence, mobility, self-help skills, speech and literacy. For this study, the self-help subscale was used to estimate the degree of ability, and responses to items on mobility, speech, reading, writing and counting were used to further describe the groups. The Wessex Scale has no published validity data but evidences modest inter-rater reliability at subscale level for both children and adults (mean kappa value of 0.62 and 0.54 for overall classification and item level reliability, respectively) $[38,39]$. The Wessex has been argued to be an effective tool for large-scale questionnaire studies [39].

The Mood Interest and Pleasure Questionnaire-Short form (MIPQ-S) [40] assesses affect and comprises 12 items, forming two subscales: mood and interest and pleasure. The measure has good internal consistency (Cronbach's alpha coefficients: total $=0.88, \operatorname{mood}=0.79$, interest and pleasure $=0.87)$, test-retest $(0.97)$ and interrater reliability (0.85). Internal consistency for subscales is good (alpha coefficient range for subscales 0.84-0.94). Concurrent validity between the MIPQ and the Aberrant Behavior Checklists's $(\mathrm{ABC})$ ranged from medium to strong $(0.36-0.73 ; p<0.001)$.

The Activity Questionnaire (TAQ) [41] was included to assess behaviours indicative of overactivity and impulsivity. The measure has 18 items which form three subscales of overactivity, impulsivity and impulsive speech. The TAQ has no published validity data but evidences item-level inter-rater reliability from 0.31 to 0.75 (mean
$0.56)$ and test-retest reliability ranges from 0.60 to 0.90 (mean 0.75). Inter-rater and test-retest reliability indices for subscales and total score exceed 0.70. Internal consistency for the subscales is good (alpha coefficient range for subscales $0.67-0.94$ ).

The Social Communication Questionnaire (SCQ) [37] was included to assess ASD behaviours. The SCQ was developed as a tool for screening for ASD in children and adults and is based on the Autism Diagnostic Interview [42]. The measure consists of 40 items grouped into three subscales: communication; social interaction and repetitive and stereotyped patterns of behaviours. The authors identify a cutoff score of 15 as indicative of ASD and a higher cutoff of 22 to differentiate between individuals with autism and those with other pervasive developmental disorders. The SCQ shows good concurrent validity with the Autism Diagnostic Interview and the Autism Diagnostic Observation Schedule [43]. Internal consistency is also good $(\alpha=0.90$ for the total scale). Whilst at $T_{1}$, the Lifetime Version of the Social Communication Questionnaire (SCQ) was employed [37], at $T_{2}$, the Current Version was administered as this version is recommended in order to evaluate measurement of change over time. All analyses conducted utilising the SCQ excluded item 17 ('has she/he ever injured her/himself deliberately, such as biting her/his arm or banging her/his head?') to prevent confounds in selfinjury analysis.

The Repetitive behaviour Questionnaire (RBQ) [44] comprises five subscales: stereotyped behaviour, compulsive behaviour, insistence on sameness, restricted preferences and repetitive speech. Previous examination of the psychometric properties of the RBQ [44] 
reveals good inter-rater reliability coefficients (range $0.46-0.80$ ), test-retest reliability (range $0.61-0.93$ ) and internal consistency (alpha coefficient range for subscales $0.50-0.78)$. Concurrent validity and content validity between the RBQ and the repetitive behaviour subscale of the ASQ are good $(0.6 ; p<0.001)$.

The Challenging Behaviour Questionnaire (CBQ) [45] was derived directly from the Challenging Behaviour Interview [46]. The CBQ evaluates the presence of selfinjury, physical aggression, verbal aggression, destruction of property and stereotyped behaviour in the last month. The measure also examines eight topographies of selfinjurious behaviour that were adapted from Bodfish and colleagues [47]. These items are as follows: hits self with body part, hits self against surface or object, hits self with object, bites self, pulls, rubs or scratches self, inserts finger or objects. The CBQ also asks about any other forms of self-injury the individual may show and asks the caregiver to specify what these topographies are. Items evaluating self-injury were used for the current study. Previous examination of the psychometric properties of the questionnaire has demonstrated good interrater reliability with reliability coefficients ranging from 0.61 to 0.89 [45]. Concurrent validity between the $\mathrm{CBI}$ and the Aberrant Behavior Checklists $(\mathrm{ABC})$ ranged from medium to strong $(0.19-0.68 ; p<0.050$ [46].

The order of the measures in the questionnaire pack was counterbalanced.

\section{Data analysis}

Data were tested for normality using KolmogorovSmirnov tests. Where data were not normally distributed $(p<0.05)$, logarithmic and square root transformations were applied in order to normalise the data. However, these were not successful in changing the distribution and therefore non-parametric analyses were employed. McNemar tests were conducted in order to examine the persistence and topographies of self-injury. A self-injury severity score was calculated by summing items regarding the length of time self-injury was displayed for, the frequency of self-injury, and the level of intervention required for self-injury. The Wilcoxon signed ranks test was used to evaluate differences in this score between $\mathrm{T}_{1}$ and $\mathrm{T}_{2}$.

Chi-square, relative risk statistics and Mann-Whitney $U$ tests were conducted in order to examine the difference between those who engaged in self-injury and those who did not on a variety of demographic and behavioural characteristics. Kruskal Wallis tests were employed to test for differences in $T_{1}$ putative risk markers between absent (self-injury absent at both $\mathrm{T}_{1}$ and $\mathrm{T}_{2}$ ), transient (self-injury present at either $\mathrm{T}_{1}$ or $\mathrm{T}_{2}$ ) and persistent (self-injury present at both $\mathrm{T}_{1}$ and $\mathrm{T}_{2}$ ) self-injury groups. Chi-square and Fisher's exact $t$ tests were used to test for these differences in categorical data.

\section{Results}

Changes in demographic and behavioural characteristics over time

Prior to analysis, the demographic and behavioural characteristics of the sample included at $\mathrm{T}_{2}$ were compared to the demographic and behavioural characteristics of the same sample at $\mathrm{T}_{1}$. This was done in order to evaluate whether any changes had occurred in demographic and behavioural characteristics that may interact with the persistence of self-injury. Table 2 presents the demographic and behavioural characteristics of the sample included at $\mathrm{T}_{2}$ and $\mathrm{T}_{1}$. In order to test for differences between the two time points, Wilcoxon signed ranks tests were conducted

Table 2 Demographic and behavioural characteristics of the selected sample at $T_{1}$ and $T_{2}$

\begin{tabular}{|c|c|c|c|c|}
\hline \multirow[b]{2}{*}{ N } & & \multirow{2}{*}{$\begin{array}{l}T_{1} \\
67\end{array}$} & \multirow{2}{*}{$\begin{array}{l}T_{2} \\
67\end{array}$} & \multirow[t]{2}{*}{$p$ value } \\
\hline & & & & \\
\hline Age & Median (IQR) & $10.00(7.00-14.00)$ & $13.50(10.00-17.00)$ & $<0.001$ \\
\hline Self help & \% partly able/able & 89.6 & 88.1 & 1.00 \\
\hline Mobility & $\%$ mobile & 95.5 & 97.0 & 1.00 \\
\hline Speech & $\%$ verbal & 95.5 & 91.0 & 0.50 \\
\hline Vision & $\%$ normal & 97.0 & 86.6 & 0.39 \\
\hline Hearing & $\%$ normal & 98.5 & 98.5 & 1.00 \\
\hline MIPQ total score & Median (IQR) & $34.00(30.00-38.00)$ & $34.00(29.00-40.00)$ & 0.264 \\
\hline TAQ total score & Median (IQR) & $44.00(24.63-53.00)$ & $41.00(21.00-50.00)$ & 0.083 \\
\hline RBQ total score & Median (IQR) & $28.00(17.00-37.00)$ & $26.00(18.00-33.00)$ & 0.289 \\
\hline SCQ total score & Median (IQR) & $28.00(15.00-37.00)$ & $21.36(6.00-34.00)$ & $<0.001$ \\
\hline SCQ total self-injury & Median (IQR) & $30.00(18.00-37.00)$ & $25.00(11.00-34.00)$ & $<0.001$ \\
\hline SCQ total no self-injury & Median (IQR) & $26.00(15.00-35.00)$ & $17.00(6.00-30.00)$ & $<0.001$ \\
\hline
\end{tabular}

${ }^{a}$ Significant differences between the two data collection points are highlighted in italics $(p<0.01$; all tests are two tailed apart from age) 
for the continuous data, and McNemar analyses were conducted for the categorical data.

The results presented in Table 2 reveal no significant differences for demographic or behavioural characteristics between $T_{1}$ and $T_{2}$. There was a significant difference between SCQ scores at $T_{1}$ and $T_{2}$. This difference was significant for both the self-injury and non-self-injury group at $\mathrm{T}_{2}$.

\section{Persistence of self-injury, topographies of self-injury and severity of self-injury}

In order to examine the persistence, remission and incidence of self-injury, the percentage of the sample who showed selfinjurious behaviour, and the various topographies of selfinjury, was calculated (see Table 3). McNemar analysis was employed to assess the persistence of self-injury.

The results presented in Table 3 reveal no significant differences in the presence or topography of self-injury displayed at $T_{1}$ and $T_{2}$, indicating that the behaviour is persistent and stable over time. In order to evaluate the stability of the severity of self-injury, the self-injury severity score at $\mathrm{T}_{1}$ (median $=6.00$, interquartile range $=4.00-8.00)$ and the self-injury severity score at $\mathrm{T}_{2}$ (median $=5.00$, interquartile range $=4.00-7.50)$ of those with persistent self-injury were compared using Wilcoxon signed ranks test. The results revealed no significant difference between the self-injury severity scores at $\mathrm{T}_{1}$ and $\mathrm{T}_{2}(N=21, p=0.374)$. Cumulative incidence was also calculated and was found to be 7 cases per 40 individuals or $17.5 \%$ over 36.4 months. In summary, the results revealed that the presence, topography and severity of self-injury were persistent and stable over three years.

\section{Demographic and behavioural characteristics associated with self-injury at $T_{2}$}

In order to examine the behavioural correlates associated with self-injury, comparisons were made between

Table 3 Percentage (point prevalence) of participants showing remission, incidence, persistence and no self-injurious behaviour

\begin{tabular}{|c|c|c|c|c|c|c|c|}
\hline Behaviour & $\begin{array}{c}\text { Absent } \\
\text { (Absent at } T_{1} \text {, } \\
\text { Absent at } T_{2} \text { ) }\end{array}$ & $\begin{array}{l}\text { Remission } \\
\text { (Present at } T_{1} \text {, } \\
\text { Absent at } T_{2} \text { ) }\end{array}$ & $\begin{array}{c}\text { Incidence } \\
\text { (Absent at } T_{1}, \\
\text { Present at } T_{2} \text { ) }\end{array}$ & $\begin{array}{c}\text { Persistent } \\
\text { (Present at } T_{1}, \\
\text { Present at } T_{2}\end{array}$ & $\begin{array}{c}P \\
\text { (2 tailed) }\end{array}$ & $\begin{array}{c}\text { Remission in } \\
\text { participants with } \\
\text { self-injury at } T_{1}{ }^{a}\end{array}$ & $\begin{array}{c}\text { Persistence in } \\
\text { participants with } \\
\text { self-injury at } T_{1}{ }^{a}\end{array}$ \\
\hline Self-injury & $\begin{array}{l}49.3 \\
\text { (33) }\end{array}$ & $\begin{array}{l}9.0 \\
(6)\end{array}$ & $\begin{array}{l}10.4 \\
\text { (7) }\end{array}$ & $\begin{array}{l}31.3 \\
(21)\end{array}$ & 1.00 & $\begin{array}{c}22.2 \\
(6)\end{array}$ & $\begin{array}{l}77.8 \\
(21)\end{array}$ \\
\hline Hits self with body & $\begin{array}{l}79.1 \\
(53)\end{array}$ & $\begin{array}{l}1.5 \\
\text { (1) }\end{array}$ & $\begin{array}{l}10.4 \\
\text { (7) }\end{array}$ & $\begin{array}{l}9.0 \\
(6)\end{array}$ & .07 & $\begin{array}{c}14.3 \\
\text { (1) }\end{array}$ & $\begin{array}{c}85.7 \\
(6)\end{array}$ \\
\hline Hits self against object & $\begin{array}{l}73.1 \\
(49)\end{array}$ & $\begin{array}{l}7.5 \\
(5)\end{array}$ & $\begin{array}{c}10.4 \\
\text { (7) }\end{array}$ & $\begin{array}{l}9.0 \\
(6)\end{array}$ & .77 & $\begin{array}{r}45.5 \\
(5)\end{array}$ & $\begin{array}{c}54.5 \\
(6)\end{array}$ \\
\hline Hits self with object & $\begin{array}{l}92.5 \\
(62)\end{array}$ & $\begin{array}{l}1.5 \\
(1)\end{array}$ & $\begin{array}{l}3.0 \\
(2)\end{array}$ & $\begin{array}{l}3.0 \\
(2)\end{array}$ & 1.00 & $\begin{array}{l}33.3 \\
(1)\end{array}$ & $\begin{array}{l}66.7 \\
(2)\end{array}$ \\
\hline Bites self & $\begin{array}{l}68.7 \\
(46)\end{array}$ & $\begin{array}{l}10.4 \\
(7)\end{array}$ & $\begin{array}{c}11.9 \\
(8)\end{array}$ & $\begin{array}{l}9.0 \\
(6)\end{array}$ & 1.00 & $\begin{array}{c}53.8 \\
\text { (7) }\end{array}$ & $\begin{array}{c}46.2 \\
(6)\end{array}$ \\
\hline Pulls self & $\begin{array}{l}88.1 \\
(59)\end{array}$ & $\begin{array}{l}3.0 \\
\text { (2) }\end{array}$ & $\begin{array}{l}4.5 \\
\text { (3) }\end{array}$ & $\begin{array}{l}4.5 \\
\text { (3) }\end{array}$ & 1.00 & $\begin{array}{c}40.0 \\
\text { (2) }\end{array}$ & $\begin{array}{l}60.0 \\
\text { (3) }\end{array}$ \\
\hline Rubs/scratches self & $\begin{array}{l}79.1 \\
(53)\end{array}$ & $\begin{array}{l}9.0 \\
(6)\end{array}$ & $\begin{array}{c}10.4 \\
\text { (7) }\end{array}$ & $\begin{array}{l}1.5 \\
\text { (1) }\end{array}$ & 1.00 & $\begin{array}{r}85.7 \\
(6)\end{array}$ & $\begin{array}{c}14.3 \\
\text { (1) }\end{array}$ \\
\hline Inserts & $\begin{array}{l}95.5 \\
(64)\end{array}$ & $\begin{array}{l}1.5 \\
\text { (1) }\end{array}$ & $\begin{array}{l}1.5 \\
\text { (1) }\end{array}$ & $\begin{array}{l}1.5 \\
\text { (1) }\end{array}$ & 1.00 & $\begin{array}{r}50.0 \\
\text { (1) }\end{array}$ & $\begin{array}{r}50.0 \\
\text { (1) }\end{array}$ \\
\hline
\end{tabular}

${ }^{\mathrm{a}}$ Remission and persistence of self-injurious behaviour in participants showing the behaviour at $\mathrm{T}_{1}$ (right of the bold line) 
those who displayed self-injury and those who did not at $\mathrm{T}_{2}$ on a variety of demographic and behavioural characteristics. Table 4 reports the differences between those with self-injury and those without on demographic measures.

The results reveal that individuals with self-injury were significantly more likely to be non-verbal than those who did not engage in self-injury. Additionally, individuals with self-injury were significantly more likely to be classified as 'not able' by the Wessex, as evidenced by poorer self-help skills. There were no significant differences between those who engaged in self-injury and those who did not, on any other demographic items.

Table 5 reports the differences between those with self-injury and those without, on measures of behavioural characteristics. Cliff's dominance (or $d$ ) statistic [48] was used to calculate effect sizes for Mann-Whitney $U$ tests.

The results in Table 5 reveal that at $\mathrm{T}_{2}$, individuals with self-injury evidenced significantly lower mood and the difference in interest and pleasure approached significance. At $T_{2}$, individuals with self-injury also evidenced significantly higher scores for measures of stereotyped behaviour, compulsive behaviour, insistence on sameness, overactivity and impulsivity. Additionally, individuals with self-injury evidenced significantly higher scores for measures of ASD phenomenology, specifically impairments in social interaction and repetitive behaviour. Small to medium effect sizes were identified for all of these differences. There were no significant differences identified for any other behavioural characteristics.

In summary, individuals with self-injury were significantly more likely to be less able and non-verbal and to show higher levels of stereotyped behaviour, compulsive behaviour, insistence on sameness, overactivity, impulsivity, repetitive behaviour and impairments in social interaction.

\section{Comparison of persistent, transient and absent self-injury} groups on $T_{1}$ behavioural and demographic variables

In order to evaluate the utility of the putative risk markers to differentiate between those with and without self-injury, participants at $\mathrm{T}_{2}$ were categorised into three self-injury groups: absent (self-injury absent at both $\mathrm{T}_{1}$ and $\mathrm{T}_{2} ; N=33$, mean $(S D)$ age in years $=12.00$ (7.03), \% male $=90.9$ ), transient (self-injury present at either $\mathrm{T}_{1}$ or $\mathrm{T}_{2} ; N=13$, mean $(S D)$ age in years $=9.54$ (3.93), \% male $=84.6$ ) and persistent (self-injury present at both $\mathrm{T}_{1}$ and $\mathrm{T}_{2} ; N=21$, mean $(S D)$ age in years $=10.81$ $(4.26), \%$ male $=81.0)$. The small sample size in the transient group prevented an analysis of behavioural and demographic variables associated with the onset or remission of self-injury. In order to identify putative risk markers, comparisons were made between $T_{1}$ data for these three groups on any variables for which differences (at $p<0.05$ ) had been obtained between the self-injury and non-selfinjury samples at either $T_{1}$ or $T_{2}$.

Table 4 Demographic variables for participants with self-injury and without self-injury at $T_{2}$

\begin{tabular}{|c|c|c|c|c|c|c|}
\hline & & $\begin{array}{l}\text { Percentage of individuals with } \\
\text { self-injury ( } N \text { - point prevalence) }\end{array}$ & $\begin{array}{l}\text { Percentage of individuals without } \\
\text { self-injury ( } N \text { - point prevalence) }\end{array}$ & Chi-square & $P$ value $^{a}$ & $\begin{array}{l}\text { Relative Risk } \\
(95 \% \mathrm{Cl})\end{array}$ \\
\hline \multirow[t]{2}{*}{ Gender } & Male & $82.1(23)$ & $89.7(35)$ & $N / A^{b}$ & 0.474 & - \\
\hline & Female & $17.9(5)$ & $10.3(4)$ & & & \\
\hline \multirow[t]{3}{*}{ Age $^{c}$} & $\leq 11$ years & $39.3(11)$ & $38.5(15)^{\prime}$ & 0.590 & 0.745 & - \\
\hline & $12-18$ years & $46.4(13)$ & $38.5(15)$ & & & \\
\hline & $\geq 19$ years & $14.3(4)$ & $20.5(8)$ & & & \\
\hline \multirow[t]{2}{*}{ Ability $^{c}$} & Able/partly able & $75.0(21)$ & $97.4(38)$ & $N / A^{b}$ & 0.008 & $2.4(1.5-3.8)$ \\
\hline & Not able & $21.4(6)$ & $2.6(1)$ & & & \\
\hline \multirow[t]{2}{*}{ Speech $^{c}$} & Verbal/partly verbal & $78.6(22)$ & $100.0(39)$ & $N / A^{b}$ & 0.005 & $2.8(2.0-3.9)$ \\
\hline & Non-verbal & $17.9(5)$ & $0.0(0)$ & & & \\
\hline \multirow[t]{2}{*}{ Mobility $^{c}$} & Mobile & $92.6(26)$ & $100.0(39)$ & $N / A^{b}$ & 0.409 & - \\
\hline & Non-mobile/partly mobile & $3.6(1)$ & $0.0(0)$ & & & \\
\hline \multirow[t]{2}{*}{ Vision } & Normal & $89.3(25)$ & $84.6(33)$ & $N / A^{b}$ & 0.724 & - \\
\hline & Poor vision/blind & $10.7(3)$ & $15.4(6)$ & & & \\
\hline \multirow[t]{2}{*}{ Hearing } & Normal & $96.4(27)$ & $100.0(39)$ & $N / A^{b}$ & 0.418 & - \\
\hline & Poor hearing/deaf & $3.6(1)$ & $0.0(0)$ & & & \\
\hline
\end{tabular}

${ }^{a}$ Significant differences $(p<0.05)$ are highlighted in italics; variables for which significant differences at $p<0.05$ were obtained at $\mathrm{T}_{1}$ are set in bold (all tests are two-tailed apart from level of ability and speech)

${ }^{\mathrm{b}}$ Fisher's exact was calculated

'One case of missing data 
Table 5 Scores and statistics for affect, repetitive behaviour, activity level and autism phenomenology for participants with and without self-injury at $T_{2}$

\begin{tabular}{|c|c|c|c|c|c|}
\hline \multirow[t]{2}{*}{ Measure subscale } & \multicolumn{2}{|c|}{ Median scores (interquartile range) } & \multirow{2}{*}{$\begin{array}{l}\text { Mann-Whitney } \\
\text { U score }\end{array}$} & \multirow[t]{2}{*}{$P$ value $^{\mathrm{a}}$} & \multirow[t]{2}{*}{ Cliff's $d$} \\
\hline & With self-injury $(N=28)$ & Without self-injury $(N=39)$ & & & \\
\hline \multicolumn{6}{|l|}{ MIPQ-S } \\
\hline Mood & $18.91(15.25-21.00)$ & $21.00(18.00-23.00)$ & 401.00 & 0.032 & 0.27 \\
\hline Interest and pleasure & $13.50(10.00-16.75)$ & $17.00(12.00-19.00)$ & 420.00 & 0.054 & - \\
\hline \multicolumn{6}{|l|}{$R B Q$} \\
\hline Stereotyped behaviour & $8.00(6.00-11.75)$ & $5.00(2.00-9.00)$ & 720.50 & 0.013 & 0.32 \\
\hline Compulsive behaviour & $8.50(4.50-12.75)$ & $4.00(1.00-8.00)$ & 748.50 & 0.005 & 0.37 \\
\hline Insistence on sameness & $4.00(2.25-8.00)$ & $3.00(0.00-6.00)$ & 680.00 & 0.043 & 0.25 \\
\hline Restricted preferences ${ }^{b}$ & $6.00(2.00-8.00)$ & $5.00(2.00-7.00)$ & 426.50 & 0.331 & - \\
\hline Repetitive language ${ }^{b}$ & $7.00(3.50-9.00)$ & $4.00(0.00-8.00)$ & 496.50 & 0.060 & - \\
\hline \multicolumn{6}{|l|}{ TAQ } \\
\hline Overactivity & $22.43(13.00-30.75)$ & $14.00(6.00-21.00)$ & 759.00 & 0.004 & 0.39 \\
\hline Impulsivity & $20.50(16.00-22.00)$ & $16.00(9.00-19.00)$ & 745.00 & 0.006 & 0.36 \\
\hline Impulsive speech ${ }^{b}$ & $3.00(1.00-8.50)$ & $5.00(2.00-6.00)$ & 383.50 & 0.403 & - \\
\hline \multicolumn{6}{|l|}{$S C Q$} \\
\hline Communication & $8.00(6.00-9.75)$ & $7.00(6.00-9.00)$ & 631.00 & 0.097 & - \\
\hline Social Interaction & $7.00(5.00-7.00)$ & $5.00(3.00-6.00)$ & 814.50 & $<0.001$ & 0.49 \\
\hline Repetitive behaviour & $9.50(7.00-12.00)$ & $5.00(3.00-9.00)$ & 825.00 & $<0.001$ & 0.51 \\
\hline
\end{tabular}

${ }^{a}$ Text in italics indicates a significant difference $\left(p<0.05\right.$, one tailed); variables for which significant differences at $p<0.05$ were obtained at $T_{1}$ are in bold ${ }^{b}$ Subscales only calculated for verbal participants

Fisher's exact tests revealed that there were no significant differences between the three groups at $T_{1}$ for speech $(p=0.059)$ or levels of ability $(p=0.171)$. Figure 1 displays the median, maximum and minimum scores and significant differences between groups on measures of behavioural characteristics.

The results reveal that across the majority of subscales for behavioural correlates, there was a broad trend towards the absent group having the lowest scores and the persistent group obtaining the highest scores, with the transient group falling between these two points. Significant differences between the groups were identified for Impulsivity (Kruskal-Wallis $\chi^{2}(2)=$ 7.33, $p=0.021$ and Social Interaction (Kruskal-Wallis $\chi^{2}$ $(2)=7.49, p=0.026$ ). In both cases, pairwise post hoc tests revealed significant differences between scores in the absent and persistent self-injury groups.

\section{Discussion}

The persistence of the presence, topography and severity of self-injury in individuals with ASD was evaluated in this study. Additionally, behavioural and demographic variables associated with persistent self-injury in ASD were delineated. The recruitment of a demographically representative sample at $\mathrm{T}_{2}$, and the utilisation of standardised measures, strengthens the validity of the study. The use of an ASD screen at $\mathrm{T}_{1}$ increases the external validity of the study and ensures robust results were obtained from the sample. This study provided the first longitudinal assessment of behavioural differences between clearly differentiated groups with absent, transient and persistent self-injury and therefore the findings have significant clinical utility. The results of the study revealed self-injury to be persistent in presence, topography and severity in individuals with ASD. The demographic and behavioural variables associated with the presence of self-injury at $T_{1}$ were revealed to be associated with self-injury at $\mathrm{T}_{2}$. Individuals with persistent self-injury evidenced significantly higher levels of impulsivity and impairments in social interaction at $\mathrm{T}_{1}$ compared to those with absent self-injury.

The results of this study revealed that self-injury was persistent over 3 years in $77.8 \%$ of those who showed self-injury at $\mathrm{T}_{1}$. This finding supports data collected in populations with intellectual disability, where the persistence of self-injury has been reported at between 71 and $84 \%[22,23]$. The results in this study differ from those reported in populations with ASD where self-injury was found to decrease significantly over time $[5,28]$. This difference is likely due to the fact that samples assessed in previous research studies were recruited from clinical services and therefore more likely to receive interventions to reduce self-injury and/or were young samples where self-injury had not yet become established. The 


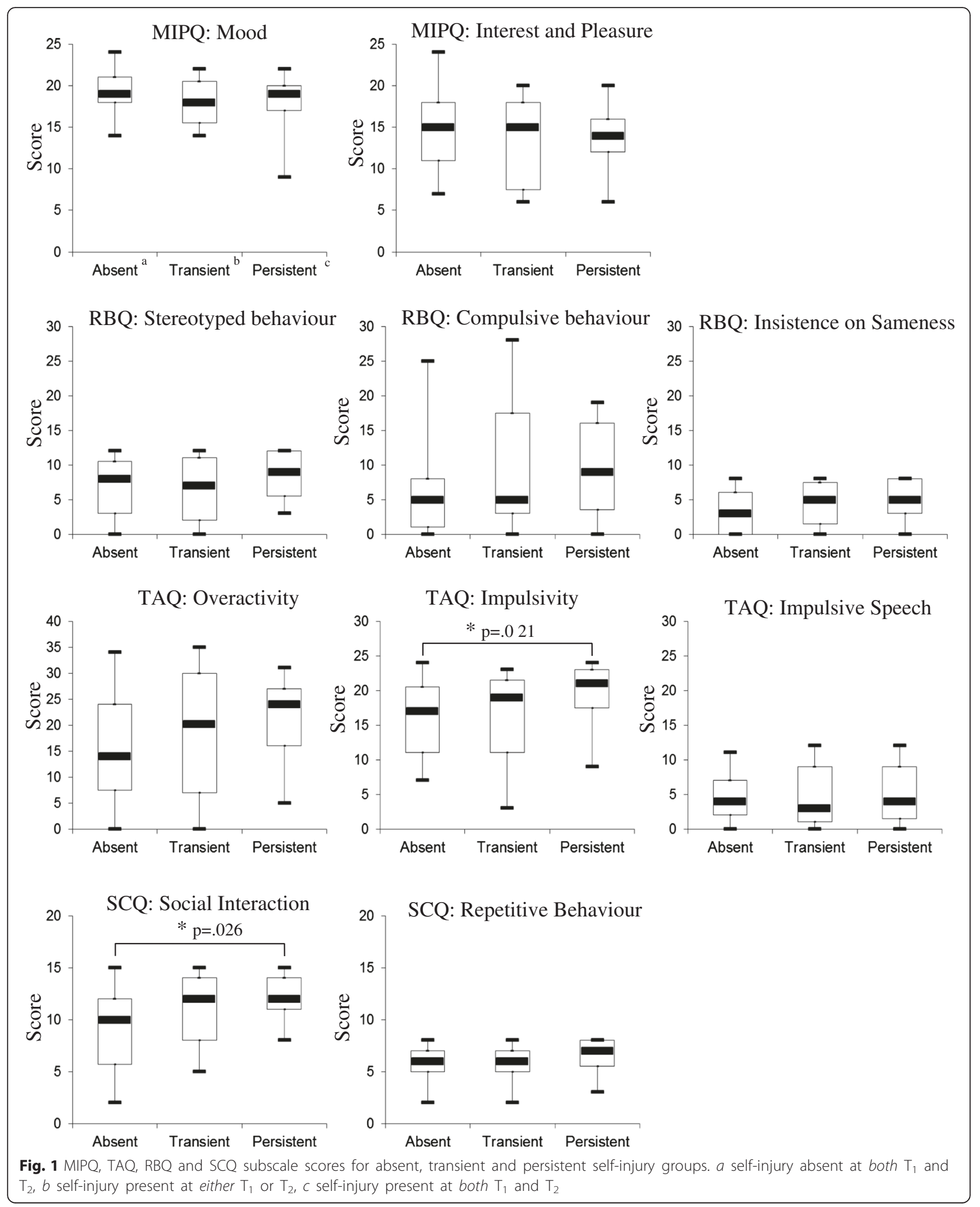

results from this study indicate that self-injury in ASD is persistent and stable over time, suggesting that intervention with younger children, where self-injury has a shorter reinforcement history and is less physically damaging during intervention, may be beneficial as the behaviour is unlikely to stop or decrease with time. The 
results extend previous research in populations with ASD by demonstrating that the topographies of selfinjury in this sample were also persistent across time. This may suggest that once established, specific forms or topographies of self-injury do not change substantially over time. However, the difference in 'hitting self with body' did approach significance and this topography was also the most persistent form of self-injury. Further research in a larger sample is warranted to investigate whether this topography of behaviour may provide an indication that self-injury is likely to persist. Interestingly, the severity of self-injury was found to be stable across time, indicating that although self-injury did not improve, it also did not increase in parameters of severity. However, these results must be interpreted with caution. Although this sample was not drawn from a clinical population, the sample was recruited from a parent support group. It is plausible that the families included may have been receiving greater levels of behavioural support and advice than families not enrolled in a support group.

The results also revealed that the majority of variables that were associated with the presence of self-injury at $\mathrm{T}_{1}$ were also associated with self-injury at $\mathrm{T}_{2}$. Being non-verbal and having lower mood and higher levels of stereotyped behaviour, compulsive behaviour, insistence on samenesss, overactivity, impulsivity, impairments in social interaction and repetitive behaviour were all associated with the presence of self-injury. There were no significant changes in the total sample over time in any of these variables. The stability of these variables and their consistent association with self-injury is important for both pragmatic and theoretical reasons. Pragmatically, the results support the utility of delineating variables that separate those individuals with ASD for whom self-injury is likely to be persistent from those with absent self-injury. The preliminary results in this study indicate that there are stable variables associated with self-injury over time which might be considered putative risk markers for persistent self-injury.

The identification of these stable variables also informs theoretical models of self-injury in ASD. The consistent association between self-injury and ability (as evidenced through adaptive skills and speech) supports findings from applied behaviour analysis, where self-injury is identified as an operant functional behaviour [49]. It is plausible that self-injury is more likely to become socially maintained in individuals with lower levels of ability, where alternative communicative strategies are limited. Secondly, the results of this study demonstrate that higher levels of ASD phenomenology are consistently associated with the presence of self-injury, replicating findings from other studies [2]. This extends the idea, identified in previous research of ASD diagnosis as a risk marker for self-injury [36], to a more refined understanding that a greater presence or severity of ASD phenomenology is associated with the behaviour. As ASD severity is known to associate with severity of intellectual disability, these results may also highlight a role for intellectual functioning as a putative correlate of selfinjury in ASD. Finally, the consistent association between low mood and self-injury replicates findings from previous studies [50]. It is possible that low mood and self-injury are indicative of depression; however, there is emerging evidence that low mood and self-injury may both be underpinned by untreated pain [19]. This requires further exploration.

The results of this study provide further evidence of compromised behavioural inhibition (as evidenced through compulsive behaviour, overactivity and impulsivity) as a behavioural correlate of self-injury. Impulsive and compulsive behaviours, such as those described in this study, and seen in individuals with attention deficit hyperactivity disorder (ADHD) have been hypothesised to be underpinned by delayed development of behavioural inhibition [51]. This impaired behavioural inhibition is suggested to reduce an individuals' ability to stop a pre-potent response to a stimulus and to terminate an ongoing response. The growing evidence associating overactivity, impulsivity, compulsive behaviours and selfinjury in ASD lends support to a theory of deficits in behavioural inhibition contributing to the development and maintenance of self-injury [3]. The presence of preserved or compromised behavioural inhibition may provide a complementary explanation to that offered in the operant literature, as to why self-injurious behaviours in young typically developing children drop out of their behavioural repertoire, whereas these behaviours persist in children with intellectual disability and ASD [52]. This hypothesis requires further research as an explanatory model of self-injury and a potential avenue for interventions in self-injury. If causal associations were identified between deficits in behavioural inhibition and the development of persistent self-injury, then early intervention for self-injury could include proactive development of strategies to increase behavioural control in 'at risk' children.

The results of this study also revealed two key differences in behavioural characteristics at $\mathrm{T}_{1}$ between the absent, transient and persistent self-injury groups at $\mathrm{T}_{2}$. Individuals with persistent self-injury evidenced significantly higher levels of impulsivity and difficulties in social interaction at $\mathrm{T}_{1}$. These behavioural characteristics represent important risk markers for persistent selfinjury, evident over a 3-year period, and highlight behaviours which could be evaluated further in young children to identify whether these same variables predict the emergence of self-injury. The study has demonstrated 
that self-injury is worryingly stable in both presence and severity but that it is possible to identify variables which are consistently associated with the presence of selfinjury and two key variables, of impulsivity and deficits in social interaction which are associated with persistent self-injury over time. Further research is now required, in younger and larger samples, to identify whether these behavioural risk markers predict the onset and severity of self-injurious behaviour. If they do, then the plausibility of identifying those with ASD most at risk of developing self-injury, and consequently the evidence base from which to develop an early intervention strategy, are strengthened. These data could be used to stratify the level of 'risk' an individual carries for the development of persistent self-injury, and thus proactive intervention using existing efficacious behavioural interventions could be targeted to those 'high risk' individuals at an earlier stage in the development of self-injury.

The study is limited by the relatively small sample size recruited at $T_{2}$. The small sample prevented investigation of variables associated with, and predictive of, incidence and remission of self-injury. However, the validity of the results is strengthened by the utilisation of an ASD screening measure at $\mathrm{T}_{1}$ to ensure a homogenous sample. An additional limitation of the study is the under-representation of individuals with self-injury at the $T_{2}$ data collection. It is possible that this under-representation may have biased the persistence and remission rates, and/or the associations between self-injury and the behavioural correlates, and thus may limit the external validity of the findings. However, no other behavioural or demographic variables differed between the two samples and at both time points the identified prevalence of self-injurious behaviour was in line with other robust estimates in the literature, suggesting that the sample is still representative of the wider ASD population. Similarly to previous longitudinal studies investigating persistence, this study did not collect data on pharmaceutical and behavioural treatment for self-injury in the sample over time $[5,23]$. Thus, it was not possible to evaluate the extent to which the identified persistence rate is the natural course of self-injury or a result of limited access or efficacy of interventions. Likewise, the identified remission rates and consequent prevalence of individuals with ASD who do not show self-injury may be due to the implementation of effective treatments. It would have been beneficial to collect these data to allow further understanding of the factors which effect persistence of self-injury. Future studies should seek to include assessment of intervention treatments, alongside the delineation of behavioural and demographic correlates, in order to build a more complete model of the trajectory of self-injury in individuals with ASD. These data should be collected in older children, adolescents and adults with ASD to detail the progression of self-injury in older cohorts.

In summary, the results have revealed that self-injury is a persistent and stable behaviour over 3-years in individuals with ASD and that risk markers of impulsivity and deficits in social interaction are associated with persistent self-injury over time.

\section{Conclusions}

The results of the present study demonstrate that selfinjurious behaviour in ASD is highly persistent over 3 years. The presence of impulsivity and deficits in social interaction are longitudinal risk markers for persistent self-injury in this population. These results lend support to models implicating behaviour dysregulation and ASD type' deficits in the development and maintenance of self-injury. The identification of these risk markers can be usefully applied to target early intervention approaches towards those individuals who are most likely to show persistent self-injury.

\section{Abbreviations \\ ASD: autism spectrum disorder; CBQ: Challenging Behaviour Questionnaire; MIPQ: Mood, Interest and Pleasure Questionnaire; RBQ: Repetitive Behaviour Questionnaire; SCQ: Social Communication Questionnaire; TAQ: The Activity Questionnaire.}

\section{Competing interests}

The authors declare that they have no competing interests.

\section{Authors' contributions}

CR contributed to the design of the study, collected and analysed the data and drafted the manuscript. CO and JM contributed to the design of the

study and revised the manuscript. LN was involved in the data collection. All authors read and approved the final manuscript.

\section{Acknowledgments}

Research Autism and Cerbra provided partial funding for this project. Recruitment of participants was supported by the National Autistic Society.

\section{Author details}

${ }^{1}$ Cerebra Centre for Neurodevelopmental Disorders, School of Psychology, University of Birmingham, Edgbaston B15 2TT, UK. ${ }^{2}$ Institute of Psychiatry, King's College London, London, UK.

Received: 2 November 2015 Accepted: 24 April 2016

Published online: 05 May 2016

\section{References}

1. Baghdadli A, Pascal C, Grisi S, Aussilloux C. Risk factors for self-injurious behaviours among 222 young children with autistic disorders. J Intellect Disabil Res. 2003;47:622-7.

2. Rattaz C, Michelon C, Baghdadli A. Symptom severity as a risk factor for selfinjurious behaviours in adolescents with autism spectrum disorders. J Intellect Disabil Res. 2015;59:730-41.

3. Richards C, Oliver C, Moss J, Nelson L. Self-injurious behaviour in individuals with autism spectrum disorder and intellectual disability. J Intellect Disabil Res. 2012;56:476-89.

4. Richman DM, Barnard-Brak L, Bosch A, Thompson S, Grubb L, Abby L. Predictors of self-injurious behaviour exhibited by individuals with autism spectrum disorder. J Intellect Disabil Res. 2012;57:429-39.

5. Shattuck PT, Seltzer MM, Greenberg JS, Orsmond GI, Bolt D, Kring S, et al. Change in autism symptoms and maladaptive behaviours in adolescents 
and adults with an autism spectrum disorder. J Autism Dev Disord. 2007;37: 1735-47.

6. Holden B, Gitlesen JP. A total population study of challenging behaviour in the county of Hedmark, Norway: prevalence, and risk markers. Res Dev Disabil. 2006;27:456-65.

7. Oliver C, Murphy G, Corbett JA. Self-injurious behaviour in people with mental handicap: a total population study. J Ment Defic Res. 1987;31:147-62.

8. Davies $\mathrm{L}$, Oliver $\mathrm{C}$. Age related prevalence of aggression and self-injury in persons with an intellectual disability. Res Dev Disabil. 2013;34:764-75.

9. Mandell DS. Psychiatric hospitilization among children with autism spectrum disorder. J Autism Dev Disord. 2008;38:1059-65.

10. Allen D, Lowe K, Brophy S, Moore K. Predictors of restrictive reactive strategy use in people with challenging behaviour. J Appl Res Intellect. 2009;22:159-68.

11. Beadle-Brown J, Murphy G, DiTerlizzi M. Quality of life for the Camberwell cohort. J Appl Res Intellect. 2009;22:380-90.

12. Hastings RP. Child behaviour problems and partner mental health as correlate of stress in mothers and fathers of children with autism. J Intellect Disabil Res. 2003;47:231-7.

13. Hastings RP, Brown T. Coping strategies and the impact of challenging behaviours on special educators burnout. Ment Retard. 2002;40:148-56.

14. Seltzer M, Greenberg J, Hong J, Smith L, Almeida D, Coe C, et al. Maternal cortisol levels and behavior problems in adolescents and adults with ASD. J Autism Dev Disord. 2010;40:457-69.

15. Knapp M, Comas-Herrera A, Astin J, Beecham J, Pendaries C. Intellectual disability, challenging behaviour and cost in care accommodation: what are the links? Health Soc Care Comm. 2005;13:297-306.

16. Harvey ST, Boer D, Meyer LH, Evans IM. Updating a meta-analysis of intervention research with challenging behaviour: Treatment validity and standards practice. J Intellect Dev Disabil. 2009;34:67-80.

17. Meyer LH, Evans IM. Science and practice in behavioural intervention-meaningful outcomes, research validity, and usable knowledge. J Assoc Pers Sev Handicaps. 1993;18:224-34.

18. Robertson J, Emerson E, Pinkney L, Caesar E, Felce D, Meek A, et al. Treatment and management of challenging behaviours in congregate and noncongregate community-based supported accommodation. J Intellect Disabil Res. 2005;49:63-72.

19. Oliver C, Richards C. Practitioner review: self-injurious behaviour in children with developmental delay. J Child Psychol Psychiatry. 2015;56:1042-54.

20. Richman DM. Early intervention and prevention of self-injurious behaviour exhibited by young children with developmental disabilities. J Intellect Disabil Res. 2008:52:3-17.

21. Oliver C, Hall S, Murphy G. The early development of self-injurious behaviour: evaluating the role of social reinforcement. J Intellect Disabil Res. 2005;49:591-9.

22. Taylor L, Oliver C, Murphy G. The chronicity of self-injurious behaviour: a long-term follow-up of a total population study. J Appl Res Intellect. 2011; 24:105-117.7.

23. Emerson E, Kiernan C, Alborz A, Reeves D, Mason H, Swarbrick R, et al. Predicting the persistence of severe self-injurious behaviour. Res Dev Disabil. 2001;22:67-75

24. Murphy G, Beadle-Brown J, Wing L, Gould J, Shah A, Holmes M. Chronicity of challenging behaviours in people with severe intellectual disabilities and/ or autism: a total population sample. J Autism Dev Disord. 2005;35:405-18.

25. Cooper SA, Smiley E, Allan LM, Jackson A, Finlayson J, Mantry D, et al. Adults with intellectual disabilities: prevalence, incidence and remission of selfinjurious behaviour, and related factors. J Intellect Disabil Res. 2009:53:200-16.

26. Totsika V, Hastings RP. Persistent challenging behaviour in people with an intellectual disability. Curr Opin Psychiatry. 2009;22:437-41.

27. Matson JL, Mahan S, Hess JA, Fodstad JC, Neal D. Progression of challenging behaviors in children and adolescents with autism spectrum disorders as measured by the autism spectrum disorders-problem behaviors for children (ASDPBC). Res Autism Spectr Disord. 2010;4:400-4.

28. Baghdadli A, Picot MC, Pry R, Michelon C, Burzstejn C, Lazartigues A, et al. What factors are related to a negative outcome of self-injurious behaviour during childhood in pervasive developmental disorders? J Appl Res Intellect. 2008;21:142-9.

29. Blanks RG, Moss SM, McGahan CE, Quinn MJ, Babb PJ. Effect of NHS breast screening programme on mortality from breast cancer in England and Wales, 1990-8: comparison of observed with predicted mortality. Br Med J. 2000;321:665-9.
30. Cohen $\mathrm{H}$, merine-Dickens $\mathrm{M}$, Smith T. Early intensive behavioural treatment: replication of the UCLA model in a community setting. J Dev Behav Pediatr. 2006;27:S145-55.

31. Eikeseth $\mathrm{S}$, Smith T, Jahr E, Eldevik S. Outcome for children with autism who began intensive behavioural treatment between ages 4 and 7 -a comparison controlled study. Behav Modif. 2007;31:264-78.

32. Remington B, Hastings RP, Kovshoff H, Espinosa FD, Jahr E, Brown T, et al. Early intensive behavioural intervention: outcomes for children with autism and their parents after two years. Am J Ment Retard. 2007;112:418-38.

33. Matson $J$, Rivet $T$. The effects of severity of autism and PDD-NOS symptoms on challenging behaviors in adults with intellectual disabilities. J Dev Phys Disabil. 2008;20:41-51.

34. Esbensen AJ, Seltzer MM, Lam KSL, Bodfish JW. Age related differences in restricted repetitive behaviours in autism spectrum disorders. J Autism Dev Disord. 2009;39:57-66

35. Danquah A, Limb K, Chapman M, Burke C, Flood A, Gore S, et al. An investigation of the factors predictive of continued self-injurious behaviour in an intellectual disability service. J Appl Res Intellect. 2009;22:395-9.

36. McClintock K, Hall S, Oliver C. Risk markers associated with challenging behaviours in people with intellectual disabilities: a meta-analytic study. J Intellect Disabil Res. 2003:47:405-16.

37. Berument SK, Rutter M, Lord C, Pickles A, Bailey A. Autism screening questionnaire: diagnostic validity. Br J Psychiatry. 1999;175:444-51.

38. Kushlick A, Blunden R, Cox G. Method of rating behavior characteristics for use in large-scale surveys of mental handicap. Psychol Med. 1973;3:466-78.

39. Palmer J, Jenkins J. The Wessex behavior rating system for mentally handicapped people—reliability study. J Ment Subnorm. 1982;28:88-96.

40. Ross E, Oliver C. Preliminary analysis of the psychometric properties of the mood, interest and pleasure questionnaire (MIPQ) for adults with severe and profound learning disabilities. Brit J Clin Psychol. 2003;42:81-93.

41. Burbidge C, Oliver C, Moss J, Arron K, Berg K, Furniss F, et al. The association between repetitive behaviours, impulsivity and hyperactivity in people with intellectual disability. J Intellect Disabil Res. 2010;54:1078-92.

42. Lord C, Rutter M, Lecouteur A. Autism diagnostic interview-revised-a revised version of a diagnostic interview for caregivers of individuals with possible pervasive developmental disorders. J Autism Dev Disord. 1994;24: 659-85.

43. Howlin P, Karpf J. Using the social communication questionnaire to identify 'autistic spectrum' disorders associated with other genetic conditions - findings from a study of individuals with Cohen syndrome. Autism. 2004:8:175-82.

44. Moss J, Oliver C, Arron K, Burbidge C, Berg K. The prevalence and phenomenology of repetitive behavior in genetic syndromes. J Autism Dev Disord. 2009;39:572-88.

45. Hyman P, Oliver C, Hall S. Self-injurious behaviour, self-restraint and compulsive behaviours in Cornelia de Lange syndrome. Am J Ment Retard. 2002;107:146-54.

46. Oliver C, McClintock K, Hall S, Smith M, Dagnan D, Stenfert-Kroese B. Assessing the severity of challenging behaviour: psychometric properties of the challenging behaviour interview. J Appl Res Intellect. 2003;16:53-61.

47. Bodfish JW, Crawford TW, Powell SB, Parker DE, Golden RN, Lewis MH. Compulsions in adults with mental retardation: prevalence, phenomenology, and comorbidity with stereotypy and self-injury. Am J Ment Retard. 1995;100:183-92.

48. Cliff N. Dominance statistics—ordinal analyses to answer ordinal questions. Psychol Bull. 1993;114:494-509.

49. Hanley G, Iwata BA, McCord BE. Functional analysis of problem behaviour: a review. J Appl Beh Anal. 2003;36:147-85.

50. Arron K, Oliver C, Moss J, Berg K, Burbidge C. The prevalence and phenomenology of self-injurious and aggressive behaviour in genetic syndromes. J Intellect Disabil Res. 2011:55:109-20.

51. Barkley RA. ADHD and the nature of self-control. New York, London: The Guilford Press; 1997.

52. Thelen E. Rhythmical stereotypies in normal human infants. Anim Behav. 1979:27:699-715. 